\title{
La experiencia del Hospital Clínic de Barcelona: integración Facultad de Medicina - IDIBAPS - Hospital Universitario*
}

\author{
The experience of the Hospital Clinic of Barcelona. Integration Medical \\ School-IDIBAPS- University Hospital.
}

Juan Rodés

Hospital Clínic i Provincial. Universidad de Barcelona

La investigación traslacional es una nueva disciplina que incorpora aspectos de ciencia básica e investigación clínica y, por tanto, requiere entrenamiento y recursos que no se suelen encontrar en los laboratorios o en departamentos clínicos. Es por ello que los institutos donde se combinan ambos tipos de investigación como universidades, centros de investigación básica y hospitales de alto nivel asistencial, son las instituciones ideales para realizar este tipo de investigación.

En el Hospital Clínic de Barcelona se ha producido un cambio en el transcurso de los últimos 40 años. Este cambio ha venido marcado por varios hechos, a destacar la creación de los servicios clínicos que substituyeron a las antiguas cátedras, la creación de los servicios de medicina, las becas post-residencia, los años sabáticos, la Fundación Clínic para la

* Presentación realizada en la Facultad de Medicina de la Universidad de Valladolid en ocasión de la celebración del $6^{\circ}$ Centenario de su creación

Correspondencia:

Juan Rodés. Director General

Hospital Clínic i Provincial

Universidad de Barcelona

e-mail: (jrodes@clinic.ub.es)
Investigación Biomédica y finalmente la puesta en marcha del Institut d'Investigacions Biomèdiques August Pi i Sunyer (IDIBAPS). En los últimos años es importante destacar también la reorganización del hospital en institutos y centros.

La producción científica en estos últimos años se ha mantenido en alza, tanto en número de publicaciones como del impacto de las revistas en las que se publica. EI IDIBAPS ha alcanzado unos resultados similares a los obtenidos por los mejores institutos de investigación del mundo occidental.

\section{Palabras clave: Investigación clínica, investigación básica, investigación traslacional, Institutos de investigación, Hospitales universitarios}

Translational research is a new discipline which incorporates aspects from both basic science and clinical investigation. As a result it requires training and resources which are not always available in laboratories or clinical departments. For this reason institutions which combine both clinical and basic research, such as universities, basic science centres and major hospitals, are the ideal institutions to perform this type of research.

Over the past 40 years, Hospital Clinic in Barcelona, have been significant with the most marked changes in the following areas; the creation of clinical units which replaced the former cathedrae, the availability 
of grants for post residents, sabbaticals, the Foundation Clinic for Biomedical Investigation and finally the inauguration of IDIBAPS, Institut d'investigacions Biomediques August Pi y Sunyer. During more recent years the hospital has undergone major re-organization dividing it into Institutes and Centres. The scientific production of the past few years has continued to grow, not just in the number of articles published but also the number of articles published in high ranking Journals. Results show that IDIBAPS equals some of the best research and investigational institutions in the western world.

Key Words: Clinical research, basic research, translational research, Research Institutes, University hospitals

La investigación biomédica se caracteriza por incluir un amplio abanico de líneas de actuación, que van desde la investigación fundamental o básica hasta la investigación clínica, la investigación en servicios de salud o la investigación epidemiológica. Estas actuaciones en materia de investigación pueden realizarse desde el ámbito molecular, orgánico o sistémico hasta llegar al estudio de grupos de población o al análisis del rendimiento técnico y económico del propio sistema de salud. En todos estos niveles se pueden formular diferentes preguntas de investigación que sean relevantes ${ }^{1,2}$.

\section{LA INVESTIGACIÓN TRASLACIONAL}

En los últimos 30 años la investigación biomédica ha experimentado una gran revolución gracias a la biología molecular. Este gran progreso ha culminado recientemente con la secuencia completa del genoma humano. A pesar de este gran progreso en la investigación biomédica básica hay, desafortunadamente, pocos ejemplos durante este mismo período de tiempo de la aplicación real de estos conocimientos en la práctica clínica. Estas aplicaciones se describen habitualmente como traslacionales, lo que significa el traslado del descubrimiento en el laboratorio a la clínica para el diagnóstico, tratamiento, pronóstico o prevención de las enfermedades que afectan al ser humano. Para resolver este problema tan importante todavía no se ha encontrado una fórmula válida. Es evidente que la investigación traslacional debe ser comprendida como una nueva forma de investigación que incluye la aplicación de los descubrimientos de la ciencia básica biomédica hacia la clínica y, simultáneamente, se debe considerar como la forma lógica de realizar preguntas científicas relevantes provenientes de la observación clínica ${ }^{3}$.

La investigación traslacional es una nueva disciplina que incorpora aspectos de ciencia básica e investigación clínica y, por tanto, requiere entrenamiento y recursos que no se suelen encontrar en los laboratorios o en departamentos clínicos. Además, la investigación traslacional no se puede llevar a cabo en los Departamentos Básicos de la Universidad o en los centros exclusivos de Biología Molecular, ya que estos centros no tienen una experiencia apropiada en investigación clínica al carecer de centros hospitalarios donde poder realizarla. Es por ello que los Institutos donde se combinan ambos tipos de investigación como Universidades, centros de investigación básica (en España correspondería a los Centros del Consejo Superior de Investigaciones Científicas) y hospitales de alto nivel asistencial, son las instituciones ideales para realizar este tipo de investigación ${ }^{4,5}$.

Con la combinación de ambos tipos de investigación todas las partes ganan. En primer lugar la investigación traslacional y la asistencia médica mejoran, y en segundo lugar es un estímulo para que los investigadores básicos realicen una investigación más realista que lógicamente beneficiará a corto o medio plazo la asistencia médica.

\section{MODELOS DE DESARROLLO DE LA INVESTIGACIÓN BIOMÉDICA. EL HOSPITAL CLÍNICO DE BARCELONA}

El Hospital Clínico de Barcelona es el centro hospitalario con mayor producción científica de España. Para entender cuales han sido los motivos que han facilitado que este hospital haya alcanzado este elevado nivel de investigación biomédica, similar a los hospitales de los países más desarrollados, es imprescindible conocer la evolución del centro en estos últimos 40 años.

En los años sesenta la investigación en el Hospital Clínico era prácticamente inexistente. Ello era debido a que el hospital (como ocurría en otros hospitales clínicos) estaba organizado a través de las Cátedras en las que imperaba el criterio organizativo que el Catedrático determinaba. No había relación entre las distintas cátedras, y tampoco había servicios centrales que diesen apoyo a la actividad médico-asistencial. Eran realmente Reinos de 
Taifas en los que no había orden ni concierto. Por citar algunas de las aberraciones organizativas que existían basta comentar que había seis "Servicios de Digestología, Neurología, Cardiología, etc”. En aquel entonces los médicos asistían voluntariamente, no existía un contrato laboral. La producción científica era realmente escasa y estaba basada en la publicación de casos clínicos en revistas españolas.

A los comienzos de los años setenta y gracias al gran impulso que realizaron los Profs. Cristóbal Pera y Ciril Rozman (Decano y Vicedecano de la Facultad de Medicina respectivamente) y con la ayuda del Dr. Miguel A. Asenjo, Director Técnico del Hospital, se realizó un gran cambio organizativo que permitió la creación de Servicios de Especialidad y de Centros de Apoyo a la asistencia: laboratorios, radiología, entre otros. Se habían eliminado los Reinos de Taifas y se había creado una estructura más departamental con servicios de especialidad. Se había, por tanto, modernizado la organización hospitalaria. Con esta nueva organización se facilitó de una manera extraordinaria la introducción del método científico en los servicios clínicos por lo que las publicaciones de aquel período ya tenían un nivel científico más elevado. En general eran trabajos descriptivos de series importantes de pacientes con una determinada enfermedad o síndrome que incluían aspectos diagnósticos, pronósticos y terapéuticos. Esta nueva orientación de la actividad científica ligada a la actividad asistencial permitió, paulatinamente pero de una forma constante, la publicación con mayor frecuencia de trabajos en revistas internacionales sometidas al sistema de "peer review".

La creación del Sindicato Médico del Hospital Clínico a finales de los años 70 dio un impulso considerable a la actividad investigadora, debido a dos hechos fundamentales: la creación de los Años Sabáticos y los Premios Fin de Residencia. A través de los Premios Fin de Residencia un cierto número de Residentes (25 aprox) podían dedicarse un año completo a la investigación al terminar su período de formación. Los Años Sabáticos permiten que cuatro profesionales al año pasen entre 6 y 12 meses en centros extranjeros de primer nivel para adquirir experiencia científica y/o tecnológica. Desde su instauración más de 100 médicos de nuestro hospital han tenido este privilegio, lo que ha propiciado que se establecieran relaciones estrechas y duraderas con las instituciones biomédicas más prestigiosas de EEUU, Europa y Japón. Estos vínculos también han facilitado que estos investigadores puedan tener ayudas de investigación de instituciones públicas extranjeras. No cabe la menor duda de que estos dos hechos fueron decisivos para impulsar la actividad investigadora del hospital.

A finales de la década de los años 80 se creó la Fundación Clínic para la Investigación Biomédica con el objetivo de dar apoyo a la investigación del hospital. Su creación facilitó la obtención de más proyectos competitivos de las Agencias Oficiales, tanto de España, como de Cataluña y Europa, y la ordenación racional de los ensayos clínicos. La creación de la Fundación permitió además separar los recursos asistenciales de los de investigación, supuso además un gran progreso organizativo y fue la simiente que permitió posteriormente la creación del Institut d'Investigacions Biomèdiques August Pi i Sunyer (IDIBAPS).

Todos estos antecedentes históricos ponen de manifiesto que desde hace muchos años la investigación es uno de los objetivos primordiales del Hospital Clínico. De hecho, en un estudio publicado por Jordi Camí (Figura 1) que evaluaba la producción científica de los hospitales en España entre los años 1994 a 2000, el Hospital Clínico de Barcelona estaba en primer lugar, tanto por el número de trabajos publicados en revistas indexadas en el SCI (4.074) como por las citas recibidas (42.564).
Figura 1

\section{PRODUCCIÓN CIENT ÍFICA HOSPITALES (1994 -2002)}

\begin{tabular}{|c|c|c|c|}
\hline Hospital & Docs & Citas & C/D \\
\hline H. Clinic de Barcelona & 4.070 & 42.564 & 10,46 \\
\hline H. Vall d'Hebron & 1.995 & 17.789 & 8,92 \\
\hline H. Sant Pau & 1.742 & 14.833 & 8,51 \\
\hline H. Ramón y Cajal & 1.698 & 13.630 & 8,03 \\
\hline H. La Paz & 1.643 & 8.996 & 5,48 \\
\hline H. 12 de Octubre & 1.594 & 13.329 & 8,36 \\
\hline H. de Bellvitge & 1.339 & 12.498 & 9,33 \\
\hline H. del Mar & 1.152 & 11.047 & 9,59 \\
\hline H. Clínico de San Carlos & 1.145 & 10.894 & 9,51 \\
\hline C. Na Sra. de la Concepci ón & 1.134 & 10.255 & 9,04 \\
\hline
\end{tabular}




\section{NUEVA ORGANIZACIÓN DEL HOSPITAL CLÍNICO Y PROVINCIAL}

La organización actual tiene 10 Institutos y 2 Centros, teniendo en cuenta que el modelo ha ido variando (por ejemplo el Instituto del Tórax surge de la fusión de dos Institutos en el planteamiento inicial, el de Enfermedades Cardiovasculares y el de Neumología y Cirugía Torácica) y cambiará en el futuro en función de aspectos relacionados con la evolución del conocimiento, liderazgos u otros (figuras 2 y 3 ).

La organización en Institutos y Centros no resuelve totalmente la orientación al paciente y, en algunas de las patologías donde participan servicios de distintos Institutos y Centros como pueden ser los diferentes tipos de cáncer, es necesario crear Unidades Funcionales entre miembros de distintos Servicios de uno o más Institutos o Centros.

\section{PRIMER NIVEL DE LA ESTRUCTURA ORGANIZATIVA}

El Director General cuenta con una estructura directiva de primer nivel constituida por el Gerente y el Director Médico para dirigir la Planificación Estratégica y establecer el seguimiento del Plan de
Actividad y Presupuesto anual de la institución. Las áreas de Sistemas de Información, Organización y Proyectos, Comunicación y Gestión y Contratación dependen directamente del Director General y funcionalmente del Gerente y del Director Médico.

El Gerente y el Director Médico actúan como coordinadores de las Direcciones Funcionales (Ingeniería y Servicios Generales, Logística, Servicios Económicos y Recursos Humanos en el caso del Gerente; Enfermería, Docencia, Investigación y EvaluaciónSoporte- Prevención en el caso del Director Médico). Los Directores Funcionales son staff y asumen funciones de apoyo a la Dirección General, al Gerente y al Director Médico y a los Institutos y Centros y aseguran la coordinación y homogeneidad entre éstos en aquellas funciones y procesos que sea necesario.

Los Directores de Instituto o Centro son los responsables últimos de la ejecución del Plan de actividad y Presupuesto de su unidad y dependen del Director General. Cada Instituto o Centro cuenta, además del Director que es un líder clínico, con un Responsable económico-administrativo y un Responsable de enfermería. Se mantienen los Servicios y se crea un Coordinador de Docencia y uno de Investigación para todo el Instituto o Centro $^{6}$.

\section{Figura 2}

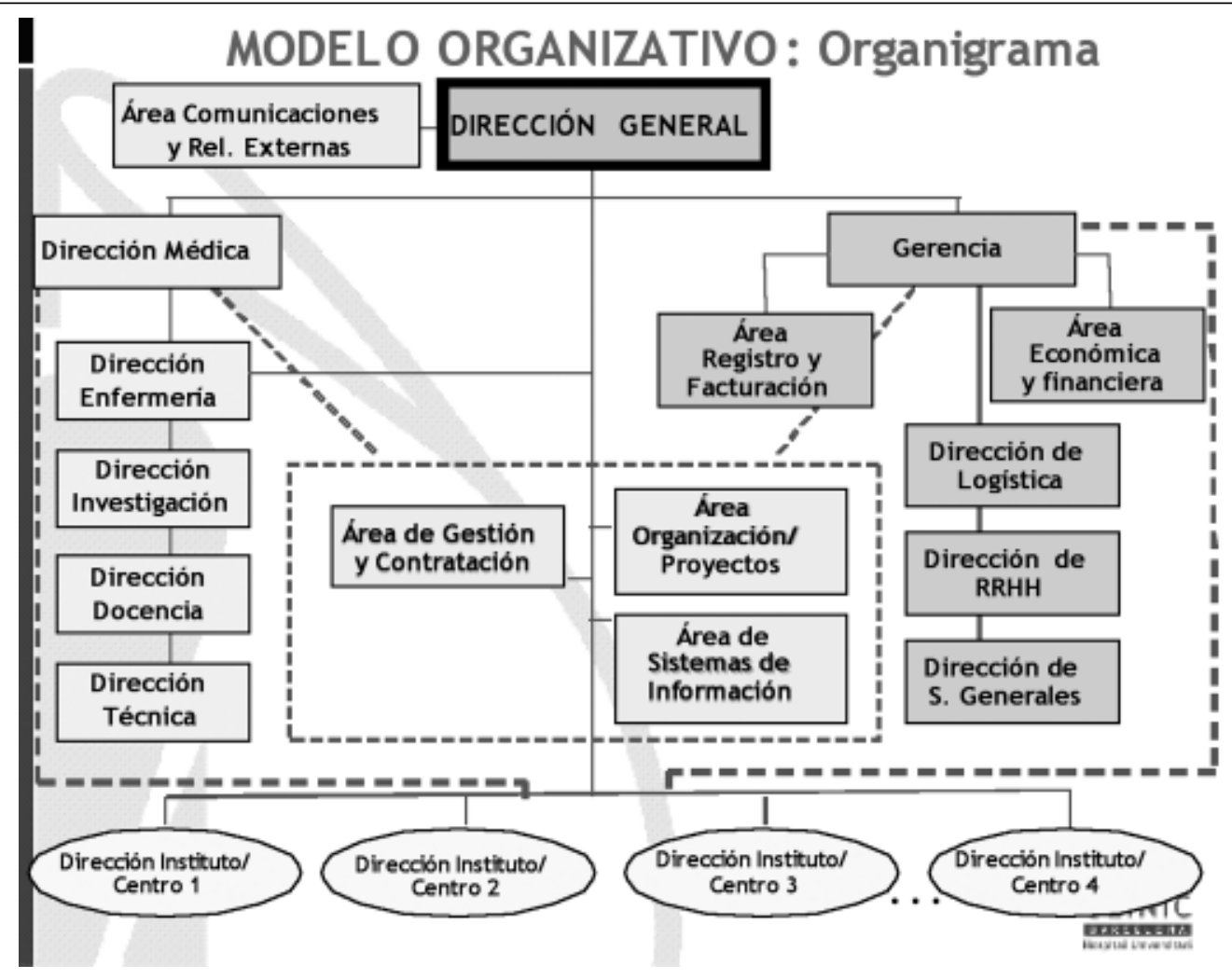


El Centro de Diagnóstico Biomédico está dirigido por un Gerente que cuenta con un Director Clínico, un Responsable de Enfermería y un Responsable Económico-administrativo.

\section{ÓRGANOS COLEGIADOS}

El Comité de Dirección General, presidido por el Director General y compuesto por el Gerente, el Director Médico y el Responsable del Área de Gestión y Contratación que actúa como secretario, da apoyo al Director General en la Planificación Estratégica, lanza y supervisa los principales proyectos de la institución y la evolución del Plan de Actividad y Presupuesto y prepara las reuniones del Comité Ejecutivo.

El Comité Ejecutivo del Hospital, presidido por el Director General y compuesto por el Gerente, el Director Médico, el Director de Recursos Humanos, el Director de Enfermería, el Responsable del Área de Gestión y Contratación y los 11 Directores de Instituto o Centro, asegura la coherencia del los planes y presupuestos de los diferentes Institutos y Centros y aprueba el Plan Asistencial, Docente y de Investigación y el Presupuesto y Plan de
Inversiones del Hospital, a propuesta del Comité de Dirección General, para su elevación a la Junta de Patronato.

Se constituyen Comités Técnicos (Comité Técnico de Gerencia, de Dirección Médica, de Dirección de Enfermería, de Dirección de Investigación, de Dirección de Docencia) para asegurar, en aquellos aspectos que sean necesarios, el desarrollo homogéneo de funciones, procesos y procedimientos entre los diferentes Institutos y Centros. Están presididos por el Director Funcional correspondiente con presencia de todos los Institutos y Centros. A modo de ejemplo, el Comité Técnico de la Dirección de Enfermería está presidido por el Director de Enfermería y compuesto por los Responsables de Enfermería de los diferentes Institutos y Centros.

Los Comités de Seguimiento de los Institutos y Centros (uno para cada Instituto o Centro) reúnen el Director General, el Gerente, el Director Médico, el Director de Recursos Humanos, el Director de Enfermería y el Responsable del Área de Gestión y Contratación del Hospital con el trío directivo de cada Instituto o Centro para aprobar los objetivos anuales de actividad y económicos anuales del Instituto o Centro y establecer un seguimiento cuatrimestral de los mismos ${ }^{7}$.

Figura 3

\section{MODELO ORGANIZATIVO: Modelo Asistencial}

\begin{tabular}{|c|c|c|c|}
\hline $\begin{array}{c}\text { Instituto de } \\
\text { Enfermedades } \\
\text { Digestivas } \\
\text { y Metabolismo }\end{array}$ & $\begin{array}{c}\text { Instituto de } \\
\text { Neurociencias }\end{array}$ & $\begin{array}{c}\text { Instituto } \\
\text { Instituto de } \\
\text { Oftalmolog ia }\end{array}$ & $\begin{array}{c}\text { Insecialidades } \\
\text { Médico } \\
\text { Quir úrgicas }\end{array}$ \\
\hline \hline $\begin{array}{c}\text { Instituto de } \\
\text { Nefrolog ia i } \\
\text { Urologia }\end{array}$ & $\begin{array}{c}\text { Direcci on M édica } \\
\text { (Urgencias, Área Quir úrgica, UASP, } \\
\text { Farma acia) }\end{array}$ & $\begin{array}{c}\text { Instituto de } \\
\text { Medicina y } \\
\text { Dermatolog ia }\end{array}$ \\
\hline \hline $\begin{array}{l}\text { Instituto } \\
\text { del Tórax }\end{array}$ & $\begin{array}{c}\text { Instituto de } \\
\text { Obstetricia, } \\
\text { Ginecolog ia } \\
\text { y Neonatolog ia }\end{array}$ & $\begin{array}{c}\text { Instituto } \\
\text { de Enfermedades } \\
\text { Hemato - } \\
\text { Oncol óqicas }\end{array}$ \\
\hline \hline Centro de Diagn óstico Biom édico & Centro de Diagn óstico por la Imagen \\
\hline
\end{tabular}




\section{INSTITUT D'INVESTIGACIONS BIOMĖDIQUES AUGUST PI I SUNYER (IDIBAPS)}

La investigación biomédica en el Hospital Clínico carecía de investigadores básicos en número suficiente para que hubiera una masa crítica apropiada. Por ello en 1993 se fundó el IDIBAPS, que es un Consorcio formado por la Generalitat de Catalunya, el Hospital Clínico y Provincial, la Universidad de Barcelona y al que también se adscribió el Instituto de Investigaciones Biomédicas de Barcelona del Consejo Superior de Investigaciones Científicas (CSIC). El objetivo científico fundamental del IDIBAPS consistió desde su inicio en la integración de la investigación clínica de calidad contrastada con la investigación básica del más alto nivel posible. El IDIBAPS tiene cinco áreas o programas de investigación: 1) oncología y hematología, 2) hepatología, gastroenterología y metabolismo, 3) enfermedades respiratorias, cardiovasculares y nefrológicas y bioingeniería, 4) neurociencias, y 5) agresión y respuesta biológica (Figura 4).

Está regido por una Junta de Gobierno presidida por el Director General de Investigación de la Generalitat de Catalunya y en la que participan además dos miembros de cada una de las instituciones que forman el consorcio, además del Director del Instituto. Tiene además un Consejo Científico externo constituido por investigadores españoles y extranjeros de un elevado nivel. Este Consejo Científico analiza anualmente la memoria del IDIBAPS y vierte sus opiniones y sugerencias en la reunión que suele celebrarse en el mes de Octubre.

El IDIBAPS puede utilizar Servicios Generales tales como estabulario, quirófanos experimentales, biblioteca, servicios audiovisuales y comunicaciones, sistemas informáticos, servicios científico-técnicos como análisis y procesos de imágenes, unidad de genómica, unidad de proteómica, unidad de bioinformática, unidad de DNA y aminoácidos, criopreservación y criogenia, banco de tejidos neurológicos, banco de tejidos y tumores y servicios de apoyo a la investigación, tales como unidad de epidemiología, estadística y unidad de ensayos clínicos. Todos estos servicios pueden depender tanto de la Universidad de Barcelona, como del Hospital Clínico o del propio IDIBAPS. Con todo ello se consigue optimizar los recursos y evitar duplicidades de tecnologías que en ocasiones tienen un coste muy elevado.

El IDIBAPS tiene 58 equipos, acreditados por la Agencia Nacional de Evaluación y Prospectiva (ANEP), de los que forman parte unos 300 investigadores, unos 300 becarios y personal de apoyo y técnico. Constituye, por tanto, una masa crítica de investigadores para poder seguir siendo considerado uno de los Institutos de investigación biomédica más potentes del país. Dentro de las actividades financiadas por el sector privado cabe destacar que el Hospital Clínico ha participado en 235 ensayos

\section{Figura 4}

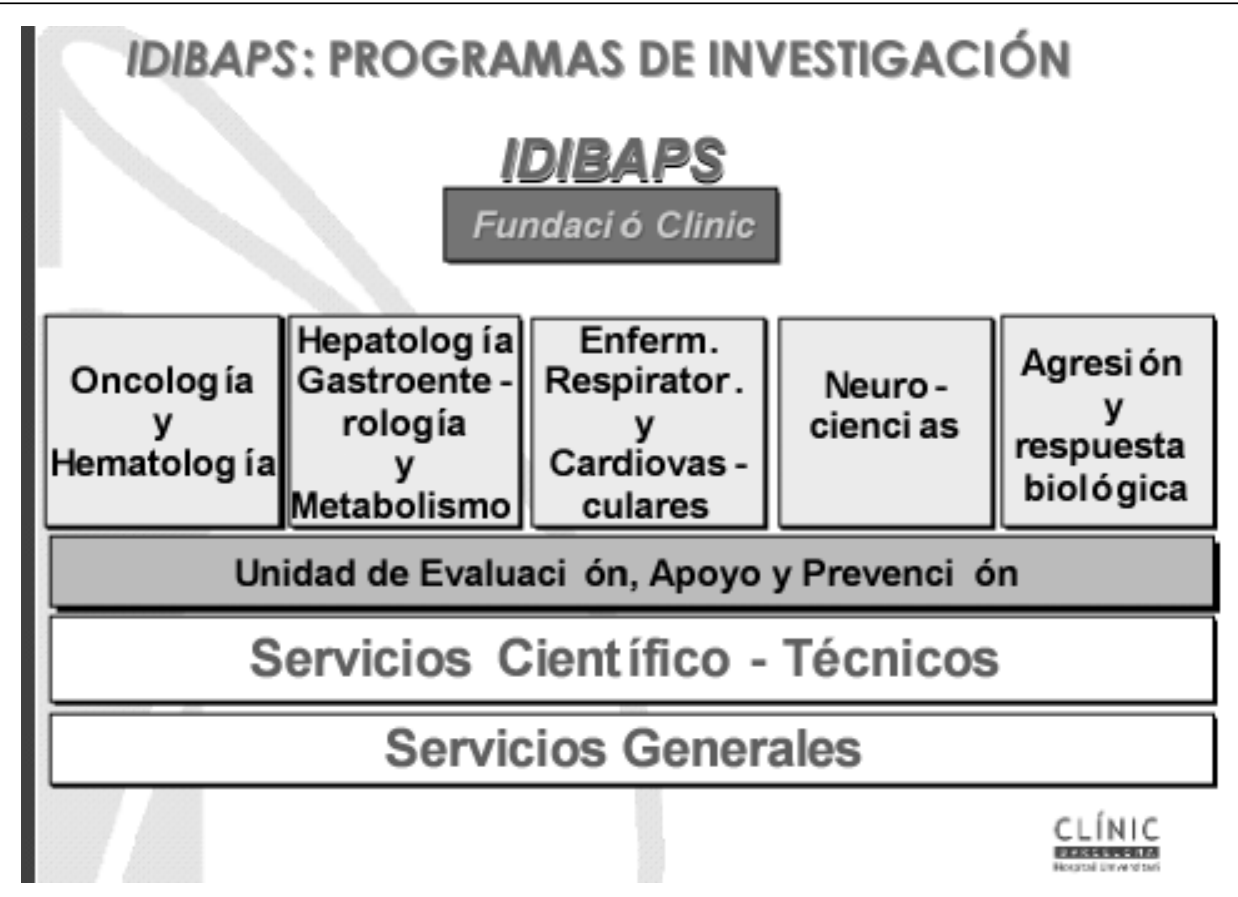




\section{Figura 5}

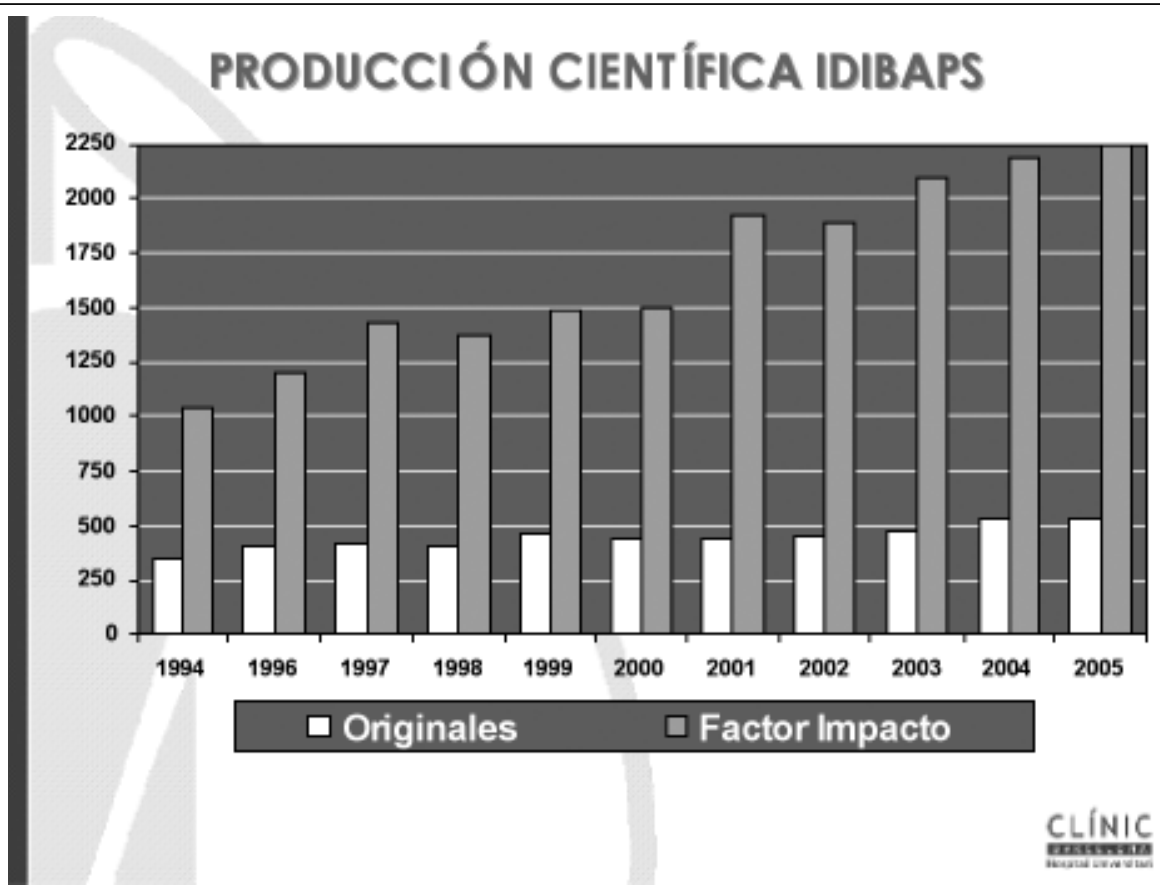

clínicos fase 1, 2 y 3 durante el año 2005. Asimismo la producción científica es muy elevada y ya ha sobrepasado la cifra de 500 artículos originales con un factor de impacto de las revistas en los que ha sido publicado de 2.242 puntos (Figura 5). La financiación que han conseguido los investigadores del IDIBAPS fue en el año 2005 de mas de $33 \mathrm{M}$ de €. El $60 \%$ de esta financiación procedía del sector público y un $40 \%$ del sector privado. Cabe señalar que la Agencia Española de Cooperación Internacional (AECI) financia en parte el Centro de Salud Internacional que el Hospital Clínico tiene en Manhiça (Mozambique). Este centro está particularmente involucrado en la investigación de vacunas contra la malaria. Recientemente, gracias a la colaboración de la Fundación Bill y Melinda Gates, los investigadores de este centro, lideraros por Pedro Alonso, han obtenido resultados muy esperanzadores en la aplicación de una nueva vacuna.

En definitiva, después de 13 años de haberse creado el IDIBAPS los resultados alcanzados han sido buenos y similares a los obtenidos por otros institutos semejantes de otros países del mundo occidental. Este es a mi juicio el modelo a seguir por los hospitales universitarios de nuestro país 8 .

\section{BIBLIOGRAFÍA}

1. Rodés J, Trilla A. Formulas para la integración de la formación clínica y básica en medicina. Med Clin (Barc) 1999; 113:379-82.

2. Rodés J, Trilla A. Investigación clínica: del laboratorio al paciente. Med Clin (Barc) 2003; 121:189-191

3. Pober JS, Neuhauser CS, Pober JM. Obstacles facing translational research in academic medical centers. FASEB $J$ 2001;15: 2303-2313.

4. Minna JD, Gazdar AF. Translational research comes of age. Nature Med 1996; 2:974-975.

5. Marwik C. Scientist recall progress and promise of translational research. J Natl Cancer Inst 2001; 93:13-15.

6. Sanz G, Pomar JL. El "Instituto de Enfermedades Cardio-vasculares". Proyecto de rediseño de los Servicios de Cardiología y Cirugía del Hospital Clínic de Barcelona. Revista Española de Cardiología 1998; 51: 620-628

7. Font D, Piqué JM, Guerra F, Rodés J. La implantación de la gestión clínica en la organización hospitalaria. Medicina Clínica (enviado para su publicación)

8. Rodés J, Font D, Trilla A, Piqué JM, Gomis R. El futuro de la gestión clínica como consecuencia del progreso científico-técnico en medicina. Medicina Clínica (enviado para su publicación) 